\title{
A Coupled Chemo-Thermo-Hydro-Mechanical Constitutive Model for Porous Media
}

\author{
Zejia Liu ${ }^{1}$, Xikui $\mathrm{Li}^{2}$, and Liqun Tang ${ }^{1}$ \\ ${ }^{1}$ College of Traffic and Communications, South China University of Technology, \\ Guangzhou, 510641, P.R. China \\ zjliuascut.edu.cn \\ ${ }^{2}$ The State key Laboratory for Structural Analysis of Industrial Equipment, \\ Dalian University of Technology, Dalian, 116024,P.R. China
}

\begin{abstract}
Basing on the existing thermo-hydro-mechanical constitutive model presented in reference[1], and the chemo-mechanical constitutive model presented in reference[2], a coupled chemo-thermo-hydro-mechanical(CTHM) constitutive model of porous media is developed in the present paper. The chemoplastic and thermoplastic coupling behavior is considered in the present constitutive model. The present coupled CTHM constitutive model has been integrated into the coupled thermo-hydro-mechanical mathematical model including contaminant translation in porous media. The numerical results are illustrated to particularly emphasize the effects of the contaminant concentration and temperature to the coupled CTHM system in porous media.
\end{abstract}

Keywords: porous media, chemo-thermo-hydro-mechanical, constitutive model, coupling.

\section{Introduction}

In the recent years, the coupled chemo-thermo-hydro-mechanical(CTHM) behavior of porous media has attracted comprehensive attentions in engineering practice.

The thermo-plasticity of clay is charactered with the thermo-softening on cohesion and pre-consolidation pressure in the constitutive model. $\mathrm{Wu}^{[1]}$ developed a THM constitutive model for unsaturated clay. The concept of chemical softening was suggested by Hueckel ${ }^{[2]}$ to describe the chemo-plastic response of clay. $\mathrm{Liu}^{[3]}$ developed this model into a couple chemo-hydro-mechanical constitutive model of unsaturated clay.

The present CTHM constitutive model is developed on the basis of the CAP model for unsaturated porous media with integration of the thermal and chemical effects on the hydro-mechanical behavior into the model.

\section{The Constitutive Model and the Consistence Algorithm}

This model is composed of four yield surfaces: The State Boundary Surface ${ }^{[1,4]}$, The Critical State Line ${ }^{[1,4]}$, The Suction Increase Curve ${ }^{[1,4]}$ and The Traction Model ${ }^{[1]}$ : 


$$
\begin{gathered}
f_{1}=q^{2}+m^{2}\left(p-3 p_{s}\left(\bar{\varepsilon}^{p}, s, T, c\right)\right)\left(p+3 p_{0 c}\left(\varepsilon_{v}^{p}, s, T, c\right)\right)=0 \\
f_{2}=q+m\left(p-3 p_{s}\left(\bar{\varepsilon}^{p}, s, T, c\right)\right)=0 \\
f_{3}=s-\left[s_{0}+\langle S e\rangle^{k 1}\left(s_{I}\left(\varepsilon_{v}^{p}, \Delta T\right)-s_{0}\right)\right]=0 \\
f_{4}=p-3 \sigma_{t}=0
\end{gathered}
$$

The cohesion is written in the form:

$$
p_{s}\left(\bar{\varepsilon}^{p}, s, T, c\right)=p_{s}\left(0, T_{r}\right)+h_{p} \bar{\varepsilon}^{p}+k_{p s} s+k_{p c} c
$$

where $p_{s}\left(0, T_{r}\right)$ is the cohesion with $s=0, c=0$ and $T=T_{r} . h_{p}, k_{p s}, k_{p c}$ are the material parameters. The pre-consolidation pressure defines as:

$$
p_{0 c}\left(\varepsilon_{v}^{p}, s, T, c\right)=p_{0}\left(\varepsilon_{v}^{p}, s, T\right) \exp \left(-\alpha_{c} c\right)
$$

where $\alpha_{c}$ is a chemical-softening parameter. The preconsolidation pressure $p_{0}\left(\varepsilon_{v}^{p}, s, T\right)$ with $\mathrm{c}=0$ is still described in format of Loading-Collapse(LC) curve $\mathrm{e}^{[1,4]}$.

The strain rate is written as:

$$
\dot{\varepsilon}_{i j}=\dot{\varepsilon}_{i j}^{p}+\dot{\varepsilon}_{i j}^{m, e}+\dot{\varepsilon}_{i j}^{s, e}+\dot{\varepsilon}_{i j}^{T, e}+\dot{\varepsilon}_{i j}^{c, e}
$$

The elastic strains ${ }^{[1,2]}$ are given by:

$\kappa$ is elastic stiffness parameter for changes in suction, $\alpha_{2}$ is the thermal expansion parameter. The chemical expansion coefficient is written in positive form ${ }^{[2,3]}$ :

$$
\beta=F_{0} \beta_{0} \exp \left[\beta_{0}(1-c+\ln c)\right](1 / c-1)
$$

The plastic potential function $g$ has the same form of yield function with dilated angle. The plastic strain rate vector is defined by the non-associated flow rule:

$$
\dot{\varepsilon}_{i j}^{p}=\dot{\lambda}^{p} \frac{\partial g}{\partial \sigma_{i j}}
$$

For a specific yield surface, the consistent condition can be written as:

$$
\dot{f}=\frac{\partial f}{\partial \sigma_{i j}} \dot{\sigma}_{i j}+\frac{\partial f}{\partial \xi} \dot{\xi}=0
$$

The evolution of the internal variable is described by a hardening/softening law:

$$
\dot{\xi}=\frac{\partial \xi}{\partial \varepsilon} \dot{\varepsilon}^{p}+\frac{\partial \xi}{\partial s} \dot{s}+\frac{\partial \xi}{\partial T} \dot{T}+\frac{\partial \xi}{\partial c} \dot{c}
$$

To derive the general form of the constitutive equations, the stress can be written as: 


$$
\dot{\sigma}_{k l}=C_{k l i j}^{e}\left[\dot{\varepsilon}_{i j}-\left(\dot{\lambda}^{p} \frac{\partial g}{\partial \sigma_{i j}}+h_{i j} \dot{s}+T_{i j} \dot{T}+D_{i j}^{c} \dot{c}\right)\right]
$$

The plastic multiplier is obtained by introducing the equation (12),(13) into (11):

$$
\begin{aligned}
& \dot{\lambda}^{p}=\left[\frac{\partial f}{\partial \sigma_{k l}} C_{k l i j}^{e} \frac{\partial g}{\partial \sigma_{i j}}-\left(\frac{\partial f}{\partial \xi} \frac{\partial \xi}{\partial \varepsilon_{i j}^{p}}\right) \frac{\partial g}{\partial \sigma_{i j}}\right]^{-1}\left[\left(\frac{\partial f}{\partial \xi} \frac{\partial \xi}{\partial s}\right) \dot{s}+\left(\frac{\partial f}{\partial \xi} \frac{\partial \xi}{\partial T}\right) \dot{T}\right. \\
& \left.+\left(\frac{\partial f}{\partial \xi} \frac{\partial \xi}{\partial c}\right) \dot{c}+\frac{\partial f}{\partial \sigma_{k l}} C_{k l i j}^{e}\left[\dot{\varepsilon}_{i j}-\left(h_{i j} \dot{s}++T_{i j} \dot{T}+D_{i j}^{c} \dot{c}\right)\right]\right]
\end{aligned}
$$

The rate constitutive equations can be derived from equation (13),(14).

\section{Numerical Results}

We consider a circular tunnel of $5 \mathrm{~m}$ diameter at $220 \mathrm{~m}$ depth excavated in unsaturated clay $^{[3]} . \mathrm{F}_{0}=0.04$. The other parameters can be found in reference [1], [3].

Two cases are analyzed $\mathrm{p}_{0}{ }^{*}=1000 \mathrm{Mpa}$ and $\mathrm{p}_{0}{ }^{*}=2 \mathrm{MPa}$. Let us consider the state of the clay at time $\mathrm{t}=3^{*} 10^{8} \mathrm{~s}$. The results around the tunnel are plotted (Fig. 2-7). These distributions are quite axisymmetrical in the region around the tunnel subjected to coupling effects. In fact, the influence of the variation of in situ stresses due to gravity is much smaller than the coupling influence and is negligible on the resulting stress and strain distributions. Figure 4 indicates that the coupling effects induce an expansion of the clay, whereas neglected volume change observed without the effect of c,T (Fig. 5). Regarding shear strains, the coupling effects are quite similar in both cases, inducing a significant increase of shear strains close to the tunnel (Fig. 6,7).

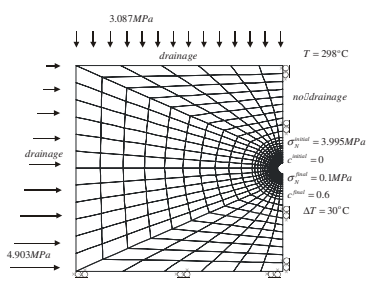

Fig. 1. The description of the problem

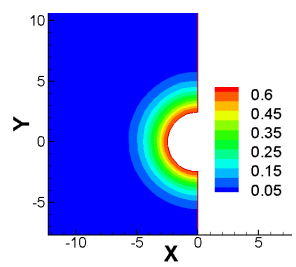

Fig. 2. Concentration

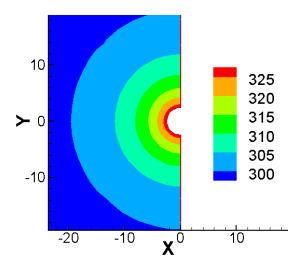

Fig. 3. Temperature
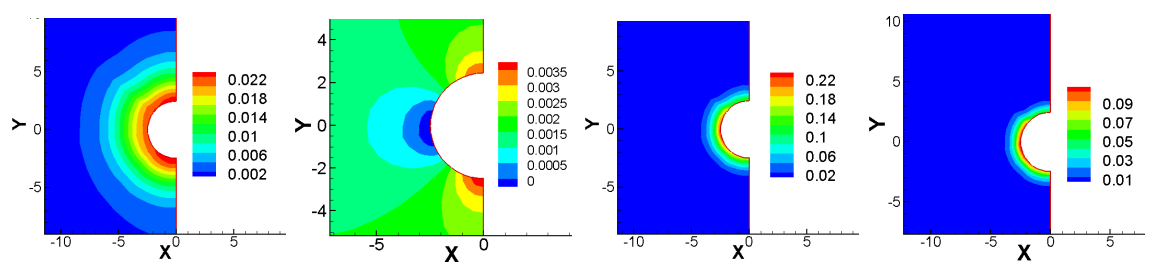

Fig. 4. $\varepsilon_{v}\left(\mathrm{p}_{0}{ }^{*}=2 \mathrm{MPa}\right)$ Fig. 5. $\varepsilon_{v}($ without $\mathrm{c}, \mathrm{T})$ Fig. 6. $\varepsilon_{e q}\left(\mathrm{p}_{0}{ }^{*}=2 \mathrm{MPa}\right)$ Fig. $7 \cdot \varepsilon_{e q}\left(\mathrm{p}_{0}{ }^{*}=1000 \mathrm{Mpa}\right)$ 


\section{Conclusions and Discussion}

The numerical results show the influence of coupling effect on the behavior around the tunnel during excavation. It is concluded that environmental load plays an important role in generating response and needs to be clearly articulated in the formulation of the problem. The proposed model may be a useful tool in designing this type of experiment, or as a matter of fact, field studies.

Acknowledgments. This work is sponsored by the National Natural Science Foundation of China under the projects No.50278012, No.10272027, No.10590354.

\section{References}

1. Wu W., et al: A thermo-hydro-mechanical constitutive model and its numerical modelling for unsaturated soils [J]. Computers and Geotechnics, 31(2)(2004) 155-167

2. Hueckel T: Chemo-plasticity of clays subjected to stress and flow of a single contaminant [J]. Int. J. Numer. Anal. Meth. in Geomech., 21(1997) 43-72

3. Liu Z., et al: Modelling chemo-hydro-mechanical behaviour of unsaturated clays: a feasibility study, Int. J. Numer. Anal. Meth. in Geomech, 29 (2005) 919-940

4. Alonso EE, Gens A, Josa A. A constitutive model for partially saturated soils [J]. Geotechnique, 40(3)(1990) 405-430 